\title{
IDENTIFICACIÓN DE PROPIEDADES DEL SUELO AGRÍCOLA EN LA PARROQUIA
} CHARAPOTÓ

\author{
AUTORES: $\quad$ María Fernanda Plúas Ponce ${ }^{1}$ \\ Fernando Ayón Villao ${ }^{2}$ \\ Jessica Morán Morán ${ }^{3}$ \\ Pedro Valdés Tamayo ${ }^{4}$ \\ William Merchán García ${ }^{5}$
}

\section{DIRECCIÓN PARA CORRESPONDENCIA: vera-raquel@unesum.edu.ec}

Fecha de recepción: 10/11/2021

Fecha de aceptación: 02/01/2022

\section{RESUMEN}

El proyecto integrador del saber se refiere a la identificación de las propiedades del suelo agrícola de la parroquia Charapotó, este proyecto se realizó en la carrera Agropecuaria en el primer semestre con el fin de integrar las ciencias básicas y las agropecuarias para preparar a los estudiantes en el perfil agropecuario y cada materia aporta al proyecto contenidos importantes para lograr las relaciones interdisciplinarias entre las asignaturas, donde el estudiante va a adquirir conocimientos referentes a las propiedades físicas y químicas que poseen los suelos agrícolas y su importancia. El objetivo del trabajo es identificar las propiedades físicas y químicas de los suelos agrícolas de la parroquia. Una vez realizado el estudio, tenemos que se destaca por tener un cuidado en sus suelos y sus productos como (arroz, maíz, cereal) además sus condiciones climatológicas son adecuadas para la efectividad y productividad de sus cultivos y

\footnotetext{
${ }^{1}$ Universidad Estatal del Sur de Manabí. Estudiante de la Carrera de Ingeniería Agropecuaria. Jipijapa. Manabí. Ecuador.

${ }^{2}$ Docente investigador de la carrera de Ingeniería Agropecuaria. Facultad de Ciencias Naturales y de la Agricultura. Universidad Estatal del Sur de Manabí. Jipijapa. Manabí. Ecuador. E-mail: fernando.ayon@unesum.edu.ec. ORCID: https://orcid.org/0000-0003-4772-9344.

${ }^{3}$ Docente investigadora de la carrera de Ingeniería Agropecuaria. Facultad de Ciencias Naturales y de la Agricultura. Universidad Estatal del Sur de Manabí. Jipijapa. Manabí. Ecuador. E-mail: jessica.moran@unesum.edu.ec. ORCID: https://orcid.org/0000-0002-6487-1038.

${ }^{4}$ Docente investigador de la Universidad Estatal del Sur de Manabí, Facultad de Ciencias Naturales y de la Agricultura, Jipijapa, Ecuador. E-mail: pedro.valdes@unesum.edu.ec. ORCID: https://orcid.org/0000-0002-7264$\underline{0440 .}$.

${ }^{5}$ Docente investigador de la carrera de Ingeniería Agropecuaria. Facultad de Ciencias Naturales y de la Agricultura. Universidad Estatal del Sur de Manabí. Jipijapa. Manabí. Ecuador. E-mail: william.merchan@unesum.edu.ec. https://orcid.org/0000- 0002- 6910- 5885.
} 
María Plúas, Fernando Ayón, Jessica Morán, Pedro Valdés, William Merchán

cuidado de animales. La actividad económica de la parroquia es la agricultura y ganadería, donde el 49,22\% de la población vinculada a la producción, el valle se siembra todo el año porque existe el sistema de canales y ríos; mientras en la zona montañosa solo se siembra una vez al año. Las principales actividades de la población están ubicadas en la parte de los balnearios de San Jacinto y San Clemente, su economía se basa en la pesca, extracción de sal, el turismo, el comercio y la artesanía, entre ellas las atarrayas y las de objetos de conchas y caracoles en menor escala.

PALABRAS CLAVE: proyecto; saberes; agricultura; pesca y economía.

\section{IDENTIFICATION OF AGRICULTURAL LAND PROPERTIES IN THE CHARAPOTÓ PARISH}

\section{ABSTRACT}

The integrating knowledge project refers to the identification of the properties of the agricultural land of the Charapotó parish, this project was carried out in the Agricultural career in the first semester in order to integrate basic and agricultural sciences to prepare students in The agricultural profile and each subject provides the project with important content to achieve interdisciplinary relationships between subjects, where the student will acquire knowledge regarding the physical and chemical properties of agricultural soils and their importance. The objective of the work is to identify the physical and chemical properties of the agricultural soils of the parish. Once the study has been carried out, we have to stand out for taking care of its soils and its products such as (rice, corn, cereal) in addition to its weather conditions are suitable for the effectiveness and productivity of its crops and animal care The economic activity of the parish is agriculture and livestock, where $49.22 \%$ of the population linked to production, the valley is sown all year round because there is a system of canals and rivers; while in the mountainous area it is only sown once a year. The main activities of the population are located in the part of the spas of San Jacinto and San Clemente, its economy is based on fishing, salt extraction, tourism, trade and crafts, including cast nets and objects of shells and snails on a smaller scale.

KEYWORDS: project; knowledge; agriculture; fishing and economy.

\section{INTRODUCCIÓN}

El proyecto integrador del saber se refiere a la identificación de las propiedades del suelo agrícola de la parroquia Charapotó, este proyecto se realiza en la carrera Agropecuaria en todos los semestres con el fin de integrar las ciencias básicas y las agropecuarias para preparar a los estudiantes en el perfil agropecuario y cada materia aporta al proyecto contenidos importantes para lograr las relaciones interdisciplinarias entre las asignaturas, en este caso se desarrolló en el primer semestre de la carrera, donde el estudiante va a adquirir conocimientos referentes a las propiedades físicas y químicas que poseen los suelos agrícolas y su importancia.

Hablar de la historia de Charapotó es hablar de su desenvolvimiento a través de las etapas de la vida desde la época aborigen; donde este territorio se lo denominó Charapotó, nombre que pasó por una metamorfosis denominativa. Al inicio se lo denominó Japoto por su ancestro aborigen, más tarde Carapoto por la influencia de los Caras al arribo de la hermosa Bahía de Caráquez.Luego se denominó Charapotó, término aborigen que contiene 2 acepciones: Chara 
criadero, Poto calabaza. En resumen, terreno propicio para el cultivo de calabaza. Esto nos demuestra que aquí existió un asentamiento humano bien organizado y preparado para la subsistencia y defensa de su territorio y de su ancestro. (PDOT, 2015)

Lo antes expuesto no da una afirmación histórica por el descubrimiento de la ciudad perdida o cuidad de lo japotoes encontrado en las proximidades al balneario de San Jacinto y en la actualidad declarado "Patrimonio intocable e histórico del cantón Sucre y especialmente de Charapotó". La Parroquia Charapotó fue fundada el 21 de septiembre de 1534, su cabecera parroquial es Charapotó. (PDOT, 2015)

La actividad económica de la parroquia es la agricultura y la ganadería en primer lugar el 49,22\% de la población, proviene de la zona baja y la zona alta, la diferencia de la baja es que este valle se siembra todo el año puesto que existe el sistema de canales y ríos; mientras la zona montañosa solo se siembra una vez al año, siempre y cuando se tenga un invierno favorable, por lo analizado es necesario la construcción de sistemas de canales de riego para esta zona alta. (GAPC, 2018)

Charapotó tiene 10.000 hectáreas aproximadamente para el cultivo de los diferentes tipos de productos en las que se siembran hortalizas como melón, sandía, pepino y pimiento y cultivos bianuales como cebolla que es de suma importancia para la economía de la parroquia, ya que esta mueve una gran parte de jornales, en especial de la mujer, 800 hectáreas son dedicadas al sembrío y cultivo de maracuyá a las que se les puede dar el valor agregado para mejorar los ingresos de los productores. (PDOT, 2015)

El territorio de la parroquia rural de Charapotó presenta laderas con pendientes fuertes, relieve irregular, suelos poco profundos en las partes altas y algo profundos en las secciones bajas. Los riesgos de erosión de los suelos en la zona presentan índices moderados y altos, esto refleja el grado potencial de susceptibilidad a la erosión.

Ordenar, las decisiones estratégicas del desarrollo respecto al estudio de los componentes biofísicos; socio -cultural; asentamientos humanos; movilidad; energía y conectividad de manera que puedan identificarse en cada caso, cuáles son los problemas y potencialidades de cada componente que permitan su posterior priorización para la solución de dichos problemas, en función de las cualidades territoriales.

Por lo antes expuesto, el objetivo del trabajo es identificar las propiedades físicas y químicas de los suelos agrícolas de la parroquia Charapotó de Cantón Sucre, provincia de Manabí, Ecuador.

\section{DESARROLLO}

En la parroquia Charapotó su relieve es muy accidentado, presenta elevaciones que oscilan entre los 20 y 400 msnm, nombradas el Cerro Mancha de Caña cuya elevación es de 466 msnm, al noroeste se elevan montañas como: Cerro Verde y Punta de Charapotó, al noreste Loma La Seca, Loma Las Pavas, Las Coronas, al sureste Loma Atravesada, Loma Las Glorias, La Laguna y al suroeste Cerro El Blanco. Se integró los cuerpos de agua y las zonas urbanas, que representan una superficie de 396,58 ha y una superficie plana intervenidas de 292,22 ha, correspondientes a las camaroneras.

Según el Instituto Espacial Ecuatoriano - IEE la geomorfología de la parroquia Charapotó se encuentra dominada por la presencia de tres unidades ambientales: 
María Plúas, Fernando Ayón, Jessica Morán, Pedro Valdés, William Merchán

a) Medio Aluvial. Se encuentran formando parte de un 16,37\% del área de la parroquia (Charapotó, Cañitas, Corre Agua, San Jacinto y San Clemente).

b) Relieves Estructurales y Colinados Terciarios: ocupan un 79,95\% del área de la parroquia, con una zona media y alta. Las unidades geomorfológicas ubicadas al oeste están asociadas a testigos de cornisa y vertientes de mesa de la Formación Borbón en las partes más altas.

c) Relieves Litorales Sedimentarios y Fluvio-Marinos: forman parte de un 0,69\% del área de la parroquia (Playas de El Pajonal, San Jacinto y San Clemente).

La actividad económica de la parroquia es la agricultura y ganadería, donde el 49,22\% de la población vinculada a la producción proviene de la zona baja y la zona alta, la diferencia de la baja, es que este valle se siembra todo el año porque existe el sistema de canales y ríos; mientras en la zona montañosa solo se siembra una vez al año, siempre y cuando se tenga un invierno favorable, por lo antes analizado es necesario la construcción de sistemas de canales de riego para la zona alta.

Las principales actividades de la población están ubicadas en la parte de los balnearios de San Jacinto y San Clemente, su economía se basa en la pesca, extracción de sal, el turismo, el comercio y la artesanía, entre ellas las atarrayas y las de objetos de conchas y caracoles en menor escala.

Charapotó tiene 10.000 hectáreas aproximadamente para el cultivo de los diferentes tipos de productos en las que se siembran hortalizas como melón, sandía, pepino y pimiento y cultivos bianuales como cebolla, que es de suma importancia para la economía de la parroquia ya que este cultivo mueve una gran parte de jornales, en especial de la mujer.800 hectáreas son dedicadas al sembrío y cultivo de maracuyá, a las que se les puede dar el valor agregado para mejorar los ingresos de los productores.

El territorio de la parroquia rural de Charapotó presenta laderas con pendientes fuertes, relieve irregular, suelos poco profundos en las partes altas y algo profundos en las secciones bajas. Los riesgos de erosión de los suelos en la zona presentan índices moderados y altos, esto refleja el grado potencial de susceptibilidad a la erosión. (GAPC, 2018)

Las principales actividades productivas de la parroquia giran en torno a la agricultura, donde en la parte baja de la parroquia el cultivo principal es el arroz, pero también se siembran cultivos de ciclo corto como es cebolla perla, pimiento, tomate, sandía, melón etc., y en la parte alta de la parroquia la producción de maíz, maracuyá, entre otros.

La parroquia Charapotó tiene grandes potenciales turísticos, su principal actividad económica es la cría y engorde de camarones en cautiverio. En la bahía o estuario del río Chone existen unas 5.000 hectáreas de piscinas dedicadas al cultivo de este crustáceo. La industria local es muy incipiente, existiendo solamente una planta procesadora y exportadora de camarones congelados, llamada Empacadora DUFER, una fábrica de hielo potable y una fábrica de agua purificada. (GAPC, 2018).

\section{Resultados y discusión}

En la parroquia Charapotó existen siete tipos de suelos en donde predominan suelos del orden de los inceptisoles + entisol con 26,57\%, seguidos de los entisoles con 24,40\%, los inseptisoles con $13,43 \%$, los ardisol + entisol con el $8,38 \%$, vertisol con el 4,41\%, mollisol 2,52\% y ardisol

18 UNESUM-Ciencias. Publicación cuatrimestral. Vol. 6, Año 2022, No. 2 (Especial Agropecuaria) 
0,0001\%. La superficie no aplicable representa el 23,29\% que son áreas urbanas y ríos. Podríamos decir entonces que el suelo de la parroquia Charapotó es muy variable y vulnerable a eventos de tipo antrópicos y naturales debido a su irregularidad. (Murillo, 2015)

En la parroquia Charapotó predomina la clase de capacidad de uso de las tierras IV con 47,80\%, seguida por la clase III con $23,53 \%$, mientras que la clase II se encuentra en menor proporción con solo el $14,12 \%$.

\section{Características de los suelos.}

Clase II: pueden ser utilizadas para el desarrollo de actividades agrícolas, pecuarias o forestales con especies adaptadas ecológicamente, incluye a suelos moderadamente profundos a profundos; sin pedregosidad, lo que permite las labores de maquinaria sin dificultad. Tierras con ligeras limitaciones, con pendientes muy suaves ( 2 a $5 \%$ ) y suaves (5 a 12\%), con erosión ligera o sin evidencia, moderamente profundos y profundos, sin pedregosidad que no limitan o imposibilitan las labores de maquinaria, con fertilidad baja a alta, tienen drenaje natural de bueno a moderado. Incluye a suelos no salinos, con toxicidad ligera o nula. No presentan periodos de inundación o éstos son muy cortos. Esta clase es apta para el desarrollo de actividades agrícolas, pecuarias o forestales con especies adaptadas ecológicamente a la zona. Su extensión es 3039,98 ha, el porcentaje del territorio parroquial es del $14,12 \%$ y la parte agrícola, pecuaria, agropecuaria o forestal con ligera limitación (Castillo, 2018).

Clase III: Son suelos con limitaciones ligeras a moderadas, se encuentran en pendientes planas (0 a $2 \%$ ), media (12 a $25 \%)$, muy suaves ( 2 a $5 \%$ ) y suaves (5 a 12\%), pueden o no presentar evidencia de erosión pudiendo ser ligera y moderada, son poco profundos a profundos, tienen poca pedregosidad, poseen fertilidad alta, mediana o baja, tienen drenaje bueno a moderado; incluyen a suelos salinos y no salinos; presentan toxicidad nula y periodos de inundación cortos, muy cortos o sin inundación. En esta clase de tierras se limita el uso de cultivos anuales, debido a que aumentan los costos de producción por el incremento de prácticas de manejo de suelo y el agua. Su extensión es 5064,69 ha y su porcentaje parroquial es $23,53 \%$, en la parte agrícola, pecuaria, agropecuaria o forestal con ligera a moderadas limitaciones.

Clase IV: Son suelos con moderadas limitaciones, se encuentran en pendientes media a fuerte (25 a $40 \%$ ); pueden o no presentar erosión actual, pudiendo ser ligera y moderada; son moderadamente profundos y no tienen pedregosidad; de textura variable y drenaje moderado a bueno. Con características no salinos; fertilidad baja, mediana y alta. No presentan periodos de inundación o éstos son muy cortos. Se restringe el establecimiento de cultivos intensivos y admite cultivos, siempre y cuando se realicen prácticas de manejo y conservación. Su extensión es 10289,76 ha y el porcentaje de la parroquia es 47,80\% agrícola, pecuaria, agropecuaria y forestal con severas limitaciones.

Clase V: son tierras con limitaciones fuertes a muy fuertes, se encuentran en pendientes entre planas y muy suaves, es decir de hasta el 5\%, suelos poco profundos con textura limosa y franco limosa y drenaje malo; fertilidad mediana; incluye suelos salinos y ligeramente salinos. Presentan periodos cortos de inundación. Los factores limitantes, determinan a esta clase no apta para cultivos o limitado para su uso específico. Su extensión es 10,30 ha y su porcentaje parroquial es 
María Plúas, Fernando Ayón, Jessica Morán, Pedro Valdés, William Merchán

0,05\%, dedicado a la parte agrícola, pecuaria, agropecuaria y forestal con ligera a moderadas limitaciones.

Clase VI: son suelos con severas limitaciones para el riego, se encuentran en pendientes fuertes (40 a 70\%) aptas para aprovechamiento de pastos, forestal y cultivos permanentes. Son moderadamente profundos, sin pedregosidad, con textura franco, drenaje bueno y fertilidad alta. Su extensión es 5,30 ha y porcentaje parroquial es de $0,02 \%$ dedicado a la parte agrícola, pecuaria, agropecuaria y forestal con muy severas limitaciones.

Clase VII: estos suelos presentan fuertes limitaciones para el laboreo, especialmente por la pendiente. Muestran condiciones para uso forestal y pastoreo con fines de conservación. Son tierras ubicadas en pendientes con suelos poco profundos; sin pedregosidad; textura variable, drenaje bueno y fertilidad de mediana a alta. No presentan periodos de inundación o éstos son muy cortos. $\mathrm{Su}$ extensión es 2270,34 ha $\mathrm{y}$ el porcentaje parroquial es de $10,55 \%$ aprovechamiento forestal

Clase VIII: son áreas que deben mantenerse con vegetación arbustiva y/o arbórea con fines de protección para evitar la erosión y mantenimiento de la vida silvestre y fuentes de agua. Son tierras con muy severas limitaciones; corresponden a pendientes planas, suaves y superficiales, sin pedregosidad y con inundabilidad permanente. Su extensión es 64,60 ha y el porcentaje parroquial es $0,30 \%$ de conservación.

Por lo antes expuesto podemos decir que la parroquia Charapotó posee un $85,45 \%$ de tierras con potencial para la agricultura y otros usos, un $10,87 \%$ de tierras para aprovechamiento forestal o con fines de conservación, debido a sus limitaciones críticas tanto a nivel topográfico y edáfico y un $0,05 \%$ de tierras de uso limitado o no adecuadas para cultivos.

La intensa dinámica de cambios de uso de suelo suscitados en la parroquia Charapotó como causa de las tendencias socioeconómicas ha reflejado la transformación del paisaje, en el que la modificación de las cubiertas de la estructura vegetal y uso de suelo son evidentes. En un análisis comparativo multitemporal se evidencia el incremento de la vegetación arbustiva y herbácea (4301 ha) y de la zona antrópica (269 ha), así mismo se observa el decrecimiento de cobertura de tierras agropecuarias (2343 ha) y Bosque (2152 ha). (PDOT, 2015).

\section{Propiedades físicas del suelo.}

Estructura del Suelo: las partículas texturales del suelo como arena, limo y arcilla se asocian para formar agregados y a unidades de mayor tamaño nombrados por peds. La estructura del suelo afecta directamente la aireación, el movimiento del agua en el suelo, la conducción térmica, el crecimiento radicular y la resistencia a la erosión. El agua es el componente elemental que afecta la estructura del suelo con mayor importancia debido a su solución y precipitación de minerales y sus efectos en el crecimiento de las plantas (Castillo, 2018).

La profundidad del suelo: la definición original del solum se denominaba como la capa superficial del suelo (horizonte A) junto con el subsuelo (E y B). El horizonte C se definía como estratos con poca formación edafogenética. De este modo la profundidad efectiva del suelo fue considerada como la espesura del suelo. Sin embargo, la presencia de raíces y la actividad biológica que frecuenta a menudo en horizonte $\mathrm{C}$ realza la importancia de incluir este horizonte en la definición de profundidad del suelo. En la práctica los estudios con levantamiento de suelos utilizan límites de profundidad arbitrarios $(200 \mathrm{~cm})$.

20 UNESUM-Ciencias. Publicación cuatrimestral. Vol. 6, Año 2022, No. 2 (Especial Agropecuaria) 
El agua almacenada o fluyente en el suelo afecta la formación del suelo, su estructura, estabilidad y erosión. El agua almacenada es el factor principal para satisfacer la demanda hídrica de las plantas.

Cuando un campo se encuentra encharcado, el espacio de aire en el suelo se desplaza por el agua. Se denomina Capacidad de Campo (CC) a la cantidad de agua el suelo es capaz de retener luego de ser saturado y dejado drenar libremente evitando evapotranspiración y hasta que el potencial hídrico se estabilice (tras 24 a 48 horas de la lluvia o riego). El agua ocupando el espacio de los poros más grandes (macroporos) drena hacia capas inferiores bajo la fuerza de gravedad. Los poros más pequeños (microporos) se llenan de agua y los más grandes de aire y agua.

El punto Capacidad de Campo corresponde a una succión de 1/3 bar. Las plantas deben producir una succión hasta 15 bares como máximo. A los 15 bares de succión la cantidad de agua en el suelo se denomina por el Punto de Marchitez Permanente (PMP). A ese punto las plantas pierden la capacidad de succión y siguen perdiendo agua mediante la transpiración. Se pierde la turgencia de la planta resultando en su marchitez. Gráficamente la diferencia entre el Punto de Capacidad de Campo y el Punto de Marchitez Permanente resulta en el agua disponible para cultivo en $\mathrm{mm}$ o expresado porcentualmente. La textura del suelo influye en la cantidad de agua en un suelo drenado hasta el punto de capacidad de campo y la cantidad que está disponible para las plantas. La humedad del suelo que se encuentra disponible se puede determinar en el laboratorio como se ilustra en las curvas de retención de humedad del suelo.

La textura del suelo se refiere a la proporción de componentes inorgánicos de diferentes formas y tamaños como arena, limo y arcilla. La textura es una propiedad importante ya que influye como factor de fertilidad y en la habilidad de retener agua, aireación, drenaje, contenido de materia orgánica y otras propiedades.

El triángulo de textura de suelos según la FAO se usa como una herramienta para clasificar la textura. Partículas del suelo que superan tamaño de $2,0 \mathrm{~mm}$ se definen como piedra y grava y también se incluyen en la clase de textura. Por ejemplo, un suelo arenoso con $20 \%$ de grava se clasifica como franco arenoso con presencia de gravas. Cuando predominan componentes orgánicos se forman suelos orgánicos en vez de minerales.

El color del suelo depende de sus componentes y varía con el contenido de humedad, materia orgánica y grado de oxidación de minerales presentes. Se puede evaluar como una medida indirecta ciertas propiedades del suelo. Se usa para distinguir las secuencias en un perfil del suelo, determinar el origen de materia parental, presencia de materia orgánica, estado de drenaje y la presencia de sales y carbonato.

La consistencia es la propiedad que define la resistencia del suelo a la deformación o ruptura que pueden aplicar sobre él. Según su contenido de humedad la consistencia del suelo puede ser dura, muy dura y suave. Se mide mediante tres niveles de humedad; aire-seco, húmedo y mojado. Para la construcción se requieren medidas más precisas de resistencia del suelo antes de la obra.

El espacio poroso del suelo se refiere al porcentaje del volumen del suelo no ocupado por sólidos. En general el volumen del suelo está constituido por $50 \%$ materiales sólidos (45\% minerales y $5 \%$ materia orgánica) y $50 \%$ de espacio poroso. Dentro del espacio poroso se pueden distinguir macro poros y micro poros donde agua, nutrientes, aire y gases pueden circular o retenerse. Los 
María Plúas, Fernando Ayón, Jessica Morán, Pedro Valdés, William Merchán

macro poros no retienen agua contra la fuerza de la gravedad, son responsables del drenaje, aireación del suelo y constituyen el espacio donde se forman las raíces. Los micro poros retienen agua y parte de la cual es disponible para las plantas.

Mediante la determinación de la densidad se puede obtener la porosidad total del suelo. Se refiere al peso por volumen del suelo. Existen dos tipos de densidad, real y aparente. La densidad real, de las partículas densas del suelo, varía con la proporción de elementos constituyendo el suelo y en general está alrededor de 2,65. Una densidad aparente alta indica un suelo compacto o tenor elevado de partículas granulares como la arena. Una densidad aparente baja no indica necesariamente un ambiente favorecido para el crecimiento de las plantas.

El agua fluye en el suelo debido a varios tipos de fuerzas como de gravedad, ascenso capilar y osmosis. Entre fuerzas de succión 0 y 1/3 bar el agua fluye en el suelo por las fuerzas de gravedad, este fenómeno se nombra por flujo saturado. Fuerzas de succión más elevadas se nombran flujos no saturados. Los flujos de agua se pueden medir en campo mediante la Conductividad Hidráulica. Se puede obtener información fundamental en la circulación del agua en el suelo mediante la descripción de suelos de las clases de drenaje y sus características asociadas (propiedades gléyicas y stágnicas).

Propiedades Químicas del suelo según la (Organización de las naciones unidas para la alimentación y la agricultura, 2018)

La Capacidad de Intercambio Catiónico (CIC) es una medida de cantidad de cargas negativas presentes en las superficies de los minerales y componentes orgánicos del suelo (arcilla, materia orgánica o sustancias húmicas) y representa la cantidad de cationes que las superficies pueden retener $(\mathrm{Ca}, \mathrm{Mg}, \mathrm{Na}, \mathrm{K}, \mathrm{NH} 4$ etc.). Estos serán intercambiados por otros cationes o iones de hidrogeno presentes en la solución del suelo y liberados por las raíces. El nivel de CIC indica la habilidad de suelos a retener cationes, disponibilidad y cantidad de nutrientes a la planta, su $\mathrm{pH}$ potencial entre otras. Un suelo con bajo CIC indica baja habilidad de retener nutrientes, arenoso o pobre en materia orgánica. La unidad de medición de CIC es en centimoles de carga por kg de suelo cmolc/kg o meq/ $100 \mathrm{~g}$ de suelo.

$\mathrm{El} \mathrm{pH}$ (potencial de hidrógeno) determina el grado de adsorción de iones $(\mathrm{H}+)$ por las partículas del suelo e indica si un suelo está acido o alcalino. Es el indicador principal en la disponibilidad de nutrientes para las plantas, influyendo en la solubilidad, movilidad, disponibilidad y de otros constituyentes y contaminantes inorgánicos presentes en el suelo. El valor del $\mathrm{pH}$ en el suelo oscila entre 3,5 (muy ácido) a 9,5 (muy alcalino). Los suelos muy ácidos $(<5,5)$ tienden presentar cantidades elevadas y tóxicas de aluminio y manganeso. Los suelos muy alcalinos $(>8,5)$ tienden a dispersarse. La actividad de los organismos del suelo es inhibida en suelos muy ácidos y para los cultivos agrícolas el valor del $\mathrm{pH}$ ideal se encuentra en 6,5.

En el suelo se encuentran los cationes ácidos (hidrógeno y aluminio) y los cationes básicos (calcio, magnesio, potasio y sodio). La fracción de los cationes básicos que ocupan posiciones en los coloides del suelo se refiere al porcentaje de saturación de bases. Cuando el pH del suelo indica 7 (estado neutral) su saturación de bases llega a un 100 por ciento y significa que no se encuentran iones de hidrógeno en los coloides. La saturación de bases se relaciona con el pH del suelo. Se utiliza únicamente para calcular la cantidad de limo requerida en un suelo acido para neutralizarlo.

22 UNESUM-Ciencias. Publicación cuatrimestral. Vol. 6, Año 2022, No. 2 (Especial Agropecuaria) 
La cantidad de nutrientes presente en el suelo determina su potencial para alimentar organismos vivos. Los 16 nutrientes esenciales para el desarrollo y crecimiento de las plantas se suelen clasificar entre macro y micro nutrientes dependiendo de su requerimiento para el desarrollo de las plantas. Los macronutrientes se requieren en grandes cantidades e incluyen Carbono(C), Hidrógeno (H), Nitrógeno(N), Fósforo (P), Potasio (K), Calcio (Ca), Magnesio (Mg), Azufre(S). Los micronutrientes por otro lado se requieren en pequeñas, su insuficiencia puede dar lugar a carencia y su exceso a toxicidad, se refieren a Hierro (Fe), Zinc (Zn), Manganeso (Mn), Boro (B), Cobre $(\mathrm{Cu})$, Molibdeno (Mo), Cloro $(\mathrm{Cl})$.

La vegetación fija el carbono de la atmosfera por fotosíntesis transportándolo a materia viva y muerta de las plantas. Los organismos del suelo descomponen esta materia transformándola a Materia Orgánica del Suelo (MOS). El carbono se libera de la biomasa para la MOS, en organismos vivos por un cierto tiempo o se vuelve a emitir para la atmosfera por respiración de los organismos (organismos del suelo y raíces) en forma de dióxido carbono, $\mathrm{CO} 2$, o metano $\mathrm{CH} 4$, en condiciones de encharcamiento en el suelo. La MOS se encuentra en diferentes grados de descomposición y se distingue en distintas fracciones como lábiles (compuestas de hidratos de carbono, ligninas, proteínas, taninos, ácidos grasos) o fracciones húmicas (ácidos fúlvicos, ácidos húmicos y huminas).Las fracciones lábiles resultan más rápidas en digerir para los microorganismos resultando en respiración de carbono y plazo de permanencia más corto en el suelo. Las fracciones húmicas se encapsulan en los agregados del suelo y son más difíciles para acceder. Además, su composición es más estable con químicos más complejos de descomponer y permanecen por periodos muy largos en el suelo.

El Carbono Orgánico del Suelo (COS) mejora las propiedades físicas del suelo, aumenta la Capacidad de Intercambio Catiónico, la retención de humedad y contribuye con estabilidad de suelos arcillosos al ayudar a aglutinar las partículas para formar agregados. La MOS está compuesta en mayoría de carbono, tiene una capacidad de retener una gran proporción de nutrientes, cationes y oligoelementos esenciales para el crecimiento de las plantas. Gracias a la MOS la lixiviación de nutrientes se inhibe y es integral a los ácidos orgánicos que disponibilizan los minerales para las plantas y regulador del $\mathrm{pH}$ del suelo. Se reconoce globalmente que el tenor de carbono orgánico en el suelo sea un factor fundamental para la salud del suelo, forma parte fundamental del ciclo de carbono y tiene gran importancia en la mitigación a los efectos del cambio climático.

\section{Nitrógeno del Suelo}

El nitrógeno del suelo es uno de los elementos de mayor importancia para la nutrición de las plantas y más ampliamente distribuido en la naturaleza. Se asimila por las plantas en forma catiónica de amonio NH4+ o aniónica de nitrato NO3-. A pesar de su amplia distribución en la naturaleza se encuentra en forma inorgánica por lo que no se pueden asimilar directamente.

Además, existen las formas gaseosas del $\mathrm{N}$ pero son muy pequeñas y difíciles de detectar como óxido nitroso (N2O), óxido nítrico (NO), dióxido de nitrógeno (NO2), amoníaco (NH3) y nitrógeno molecular presente en la atmósfera del suelo (N2).

\section{La salinización del suelo}


María Plúas, Fernando Ayón, Jessica Morán, Pedro Valdés, William Merchán

Se refiere a la acumulación de sales solubles en agua en el suelo. Las sales que se pueden encontrar en un nivel freático salino se transportan con el agua a la superficie del suelo mediante ascenso capilar y una vez que el agua se evapore se acumulan en la superficie del suelo. La salinización suele ocurrir con manejo de riego inapropiado sin tomar en consideración el drenaje e lixiviación de las sales por fuera de los suelos. Las sales también se pueden acumular naturalmente o por la intrusión de agua marina. La salinización elevada en el suelo lleva a la degradación de los suelos y la vegetación. Las sales más comunes se encuentran en combinaciones de los cationes de sodio, calcio, de magnesio y de potasio con los aniones de cloro, sulfato y carbonatos.

\section{La alcalinización del suelo}

La alcalinización, o salinidad del suelo, se define como el exceso de sodio intercambiable en el suelo. A medida que su concentración incrementa en el suelo empieza a reemplazar otros cationes. Los suelos sódicos se frecuentan en regiones áridas y semiáridas y se encuentran muchas veces inestables con propiedades físicas y químicas muy pobres. Debido a ello el suelo se encuentra impermeable disminuyendo la infiltración, percolación, infiltración del agua por el suelo y por último el crecimiento de las plantas.

\section{Contenido de carbonato de calcio en el suelo}

El carbonato de calcio, $\mathrm{CaCO} 3$, es una sal poco soluble que se encuentra naturalmente en varias formas y en varios grados de concentración en el suelo. Su presencia juega un papel fundamental en la estructura del suelo si se encuentra en concentraciones moderadas. Se utiliza como enmienda para neutralizar el pH de suelos ácidos y para suministrar el nivel de Calcio (Ca) para la nutrición de las plantas. Sin embargo, puede resultar problemático si su concentración llega a exceder la capacidad de adsorción en el suelo formando complejos insolubles con otros elementos. Estos componentes son difíciles de asimilar por las plantas llevando a su acumulación. Cantidades excesivas de calcio puede por ello restringir la disponibilidad de fósforo, boro y hierro para las plantas.

\section{Contenido de Sulfato de calcio (Yeso) en el suelo}

En los suelos puede ocurrir la presencia de la acumulación secundaria de yeso (CaSO4.2H2O) extendiéndose principalmente en regiones muy áridas o donde el lavabo del suelo esté restringido a causa de baja permeabilidad. Los suelos afectados por concentraciones elevadas de yeso se han desarrollado en gran mayoría en depósitos no consolidados aluviales, coluviales y eólicos de material meteorizado con alto contenido de bases. Existe una vaga vegetación natural que cubre los suelos con alto contenido de yeso, de hecho, se encuentran apenas arbustos y árboles xerófilos y/o hierbas efímeras. (ONUAA, 2018)

\section{Métodos de control de las enfermedades que afectan los cultivos y animales.}

Los Servicios Veterinarios nacionales (SV) están en el centro del sistema de prevención y control de las enfermedades animales. Son principalmente responsables de la detección precoz y la respuesta rápida a los brotes de enfermedades animales emergentes o reemergentes. De ahí que mejorar la gobernanza de los SV sea una prioridad mundial, ya que una legislación adecuada

garantiza y refuerza la calidad y la eficacia de los sistemas de prevención y de control de las enfermedades. En los países en vías de desarrollo y en transición, los SV necesitan más recursos

24 UNESUM-Ciencias. Publicación cuatrimestral. Vol. 6, Año 2022, No. 2 (Especial Agropecuaria) 
humanos y financieros y más capacidades con el fin de poder garantizar la sanidad animal y, por ende, la salud pública, incluida la seguridad y la inocuidad alimentaria, en todo el mundo.

El requisito de toda acción de prevención y control de las enfermedades animales es una vigilancia activa (planificada) o pasiva (basada en la detección de los acontecimientos) eficaz. La OIE define la vigilancia como «La recopilación, el cotejo y el análisis sistemáticos y continuos de datos, y la difusión rápida de la información a quienes la necesiten para tomar medidas». (Código sanitario para los animales terrestres de la OIE). Esto puede garantizarse únicamente con una campaña de concienciación que incluya y reúna al conjunto de actores en todos los niveles de la cadena de producción animal, es decir, desde el ganadero, de su veterinario y del laboratorio local, hasta las más altas autoridades veterinarias nacionales.

Un sistema para detectar e identificar de manera precoz la incursión o la emergencia / reemergencia de una enfermedad o una infección en un país, zona o compartimento es el sistema de detección de enfermedades. El sistema de detección precoz debe estar bajo el control de los Servicios Veterinarios conformes a las normas pertinentes de la OIE, y reunir las siguientes características:

- Cobertura representativa de las poblaciones de animales diana, a cargo de todos los servicios presentes en el terreno y que colaboran eficazmente con los propietarios de animales;

- Capacidad de efectuar investigaciones epidemiológicas eficaces sobre las enfermedades y de notificarlas;

- Acceso a los laboratorios capaces de diagnosticar y diferenciar las enfermedades pertinentes;

- Un programa de formación de los veterinarios, los para profesionales de veterinaria y demás personas encargadas del cuidado de los animales, para detectar y notificar incidentes zoosanitarios;

- Obligaciones legales de los veterinarios del sector privado con respecto a la autoridad veterinaria; una cadena de mando bien establecida a nivel nacional. (OMSS, 2017)

\section{Ventajas y desventajas de los pesticidas químicos}

El uso de pesticidas químicos se ha generalizado debido a su coste relativamente bajo, su facilidad de aplicación y su eficacia, disponibilidad y estabilidad. Los pesticidas químicos son, por lo general, de acción rápida, lo que limita el daño de los cultivos.

Los pesticidas químicos tienen algunas desventajas importantes, sin embargo, siguen siendo muy vendidos y utilizados. En este artículo discutiremos cuatro de las desventajas de los pesticidas químicos. En primer lugar, los pesticidas químicos, con frecuencia, no solo son tóxicos para los organismos para los que han sido concebidos, sino también para otros organismos. Los pesticidas químicos pueden subdividirse en dos grupos: pesticidas no selectivos y pesticidas selectivos. Los pesticidas no selectivos son los más peligrosos, porque matan toda clase de organismos, incluyendo especies inofensivas y útiles. Por ejemplo, hay herbicidas que matan tanto las malezas de hoja ancha como las gramíneas. Esto quiere decir que no son selectivos, ya que destruyen casi 
María Plúas, Fernando Ayón, Jessica Morán, Pedro Valdés, William Merchán

toda la vegetación. Los pesticidas selectivos tienen un alcance más restringido. Solo destruyen la plaga, la enfermedad o la maleza, sin afectar otros organismos. Como ejemplo podemos nombrar un herbicida que actúa únicamente sobre las malezas de hoja ancha, por lo que puede utilizarse en el césped, ya que no mata la hierba. Hoy en día, por lo general, se requiere una combinación de varios productos para el control de varias plagas, ya que casi todos los productos permitidos son selectivos y, por lo tanto, solo controlan un rango de plagas determinado.

Otra desventaja de los pesticidas químicos es la resistencia. Los pesticidas suelen ser eficaces solo durante un (corto) período de tiempo para un organismo en particular. Los organismos pueden llegar a desarrollar inmunidad a una determinada sustancia, perdiendo su efecto. Estos organismos mutan y se hacen resistentes, lo que resulta en la necesidad de usar otros pesticidas para su control. (Canna. (s.f.), 2014)

\section{Fertilidad del suelo}

La fertilidad del suelo es la capacidad que tiene el terreno para sustentar el crecimiento de las plantas y optimizar el rendimiento de los cultivos. Ello puede potenciarse por medio de fertilizantes orgánicos e inorgánicos que nutran el suelo. Las técnicas nucleares proporcionan datos útiles que mejoran la fertilidad del suelo y la producción de cultivos, al tiempo que reducen al mínimo el impacto medioambiental.

Promover la seguridad alimentaria y la sostenibilidad ambiental de los sistemas agrícolas requiere adoptar un enfoque integrado en la gestión de la fertilidad del suelo, que potencie al máximo la producción de cultivos y reduzca al mínimo la extracción de las reservas de nutrientes del suelo y la degradación de las propiedades físicas y químicas de este, lo que puede desembocar en la degradación de la tierra, incluida la erosión del suelo.

Esas prácticas de gestión de la fertilidad del suelo incluyen, entre otras cosas, el uso de abonos e insumos orgánicos, la aplicación de técnicas de rotación de cultivos con leguminosas y el empleo de germoplasma mejorado, así como saber cómo adaptar esas prácticas a las condiciones locales. (OIEA, 2015)

Aunque las lluvias no han sido suficientes, arroceros de comunidades de la parroquia Charapotó, en el centro de la provincia de Manabí, se sienten satisfechos por el resultado de la cosecha obtenida.

Ellos atribuyen este rendimiento al manejo de los canales de riego y drenaje que administra la Secretaría Nacional del Agua (Senagua) en esta zona, que es un extenso valle ubicado entre los cantones Sucre y Rocafuerte, donde predomina la cosecha de la gramínea.

"Estamos contentos porque hemos podido cosechar algunas hectáreas gracias a la ayuda de los canales de riego que pasan por nuestras parcelas”, expresó Pedro Moreira, agricultor del sitio Pasadero, perteneciente a Sucre. Personal del área de riego de la Senagua se traslada a las comunidades para dar asistencia técnica a los diferentes requerimientos de las poblaciones agrícolas.

Por otro lado, gran parte de los canales de drenaje y de riego se encuentran con agua suficiente para dotar a las diferentes plantaciones y parcelas de productos agrícolas a lo largo de todo este valle, caracterizado por su abundante producción de arroz y cebolla perla, señalaron fuentes de la Secretaría Nacional del Agua. (El Comercio, 2014)

26 UNESUM-Ciencias. Publicación cuatrimestral. Vol. 6, Año 2022, No. 2 (Especial Agropecuaria) 


\section{Arroceros de Charapotó aplicarán programa biotecnológico}

"Manejo Técnico del Cultivo Arroz Soca", con el programa biotecnológico "Suelo Sano Cultivo Sano", es el tema de la conferencia técnica que dictarán los especialistas Guillermo Pisco de JW Asociados y Javier Junco de Orius Biotech este viernes 11 de enero a las 15:00, en la comuna Bebedero de la parroquia Charapotó, provincia de Manabí, atendiendo a la invitación del agro empresario Freddy Cevallos.

El interés de los arroceros manabitas, surge de los sorprendentes resultados obtenidos en el ensayo técnico que se realizó en la propiedad de Wellington Ronquillo ubicada en el sector las Maravillas de Daule, (Guayas), con un rendimiento superior al arroz de siembra y a un costo muy inferior.

La cosecha del ensayo arroz soca (verano 2018) empezó a los 111 días con un rendimiento promedio de 58,6 sacas de arroz en cáscara de 213 libras por cuadra $(5,8 \mathrm{tm} /$ cuadra $=8,2 \mathrm{tm} / \mathrm{Ha})$, y a un costo de $\$ 600$.

Una ganancia representativa aún con el bajo precio del mercado interno, sin aplicar fungicida químico, conservando el suelo y mejorando el promedio anual de productividad del productor.

Los encargados de dictar la charla, destacan que el trabajo de investigación se basa en una óptima nivelación del terreno, cero quemas de la panca y la aplicación del programa biotecnológico "Suelo Sano Cultivo Sano" para aprovechar todos los residuos de cosecha y controlar las plagas y enfermedades de manera integral.

La producción de la gramínea, uno de los principales productos que mueve el comercio de Charapotó, da trabajo a cientos de jóvenes y adultos que atienden las labores de cultivo, transporte y proceso de pilado. Los agricultores poseen en promedio de una a 10 hectáreas.

El cereal es la base de la economía de las 12 comunidades de Charapotó y su cultivo puede desarrollarse todo el año gracias al sistema de riego provisto del agua que se succiona desde el cauce del río Portoviejo que, a su vez, es alimentado por la represa Poza Honda ubicada en la parte alta del cantón Santa Ana, centro oeste de la provincia. (Jw asociados, 2018)

\section{CONCLUSIONES}

Una vez realizado el estudio de la parroquia rural Charapotó, se tiene información necesaria y suficiente que permite llegar a la siguiente conclusión: El Cantón Sucre tiene una riqueza en su flora y fauna, tanto como en sus parroquias urbanas como rurales. La parroquia rural Charapotó se destaca por tener un cuidado en sus suelos y sus productos como (arroz, maíz, cereal) además sus condiciones climatológicas son adecuadas para la efectividad y productividad de sus cultivos y cuidadas de animales

Aprovechar en forma racional los recursos del suelo es necesario, para conocer las características de estos con el uso óptimo de acuerdo con su capacidad de producción. Esto significará una relocalización de la producción agropecuaria y forestal, por lo que en un período relativamente corto resultaría no solo en un aumento de la producción, sino, también en una mejor conservación de los recursos naturales. 
María Plúas, Fernando Ayón, Jessica Morán, Pedro Valdés, William Merchán

\section{REFERENCIAS BIBLIOGRÁFICAS}

Canna. (s.f.). (2014). Obtenido de Canna. En línea: http://www.canna.es/como_controlar_plagas_y_enfermedades_lo_biologico_frente_lo_químico.

Castillo, Isabel. (2018). Suelos del Ecuador: Características, Tipos, Usos. Lifeder. (3 de enero de 2018). En línea: https://www.lifeder.com/suelos-del-ecuador/.

El comercio. (2014). Una Buena Cosecha en Charapotó. (4 de junio de 2014). Obtenido del comercio: https://www.eluniverso.com/noticias/2014/06/04/nota/3054676/buena-cosecha-Charapotó .

GAPC (2018). Gad Parroquial Charapotó. Obtenido de GAD Parroquial Charapotó (3 de julio de 2018). http://charapoto.gob.ec/index.php/ct-menu-item-33/ct-menu-item-37.

Jw, Asociados. (2018). Obtenido de Jw asociados (11 de enero de 2018). http://jwasociados.com.ec/arroceros-decharapoto-estudian-aplicar-biotecnologia-agricola/

Murillo Moreira, Sandra Paola (2015). Plan de Desarrollo y Ordenamiento Territorial. Charapotó. En línea. https://odsterritorioecuador.ec/wp-content/uploads/2019/04/PDOT-Parroquia-Charapoto-2015-2019.pdf

OIEA. (2015). Organización Internacional de Energía Atómica. (3 de febrero de 2015). Obtenido de organización internacional de energía atómica: https://www.iaea.org/es/temas/mejora-de-la-fertilidad-del-suelo

OMSS. (2017). Organización Mundial de Sanidad de Salud. (3 de enero de 2017). Obtenido de Organización mundial de sanidad de salud: https://www.oie.int/fileadmin/Home/esp/Media_Center/docs/pdf/Fact_sheets/P-C ES.pdf

ONUAA. (2018). Organización de las Naciones Unidas para la Alimentación y la Agricultura. (4 de septiembre de 2018). Obtenido de Organización de las naciones unidas para la alimentación y la agricultura: http://www.fao.org/soils-portal/soil-survey/propiedades-del-suelo/propiedades-fisicas/es/

PDOT. (2015). Plan de Desarrollo y Ordenamiento Territorial. Gobierno Autónomo Descentralizado de la Parroquia Charapotó - Sucre - Manabí OCTUBRE 2015.En línea. https://odsterritorioecuador.ec/wpcontent/uploads/2019/04/PDOT-PARROQUIA-CHARAPOTO-2015-2019.pdf

28 UNESUM-Ciencias. Publicación cuatrimestral. Vol. 6, Año 2022, No. 2 (Especial Agropecuaria) 